\title{
Differential mating success in populations of seaweed flies (Coelopa frigida)
}

\author{
T. H. Day, \\ S. Miles, \\ M. D. Pilkington and \\ R. K. Butlin*
}

Department of Genetics, University of Nottingham, University Park, Nottingham, U.K.

* School of Biological Sciences, University of East Anglia, Norwich, U.K.

The reproductive success of seaweed flies (Coelopa frigida) was studied in natural populations. The sizes of females, both mothers and those not producing eggs, as well as of the males available to be fathers, were estimated from their wing lengths. The animals were also classified for their genotype at the alcohol dehydrogenase (Adh) locus-a polymorphic locus tightly associated with a large chromosomal inversion. The fathers' genotypes were inferred from the genotypes of the mother and her offspring. Males of different Adh genotypes had different reproductive success. Reproductive success of genotypes was negatively correlated with the mean sizes of males. Differences in the reproductive success of females could be accounted for by their differential egg laying capacity, and is probably not a consequence of their mating behaviour. The incidence of progeny with mixed paternity varied from 20 per cent initially, to about 65 per cent when the animals had been mating for 6 days. It appears from previous work that large males are more successful in laboratory trials, yet under natural conditions genotypes associated with large male size have low mating success. Ways of reconciling these results and their relevance to understanding the inversion polymorphisms are discussed.

\section{INTRODUCTION}

For 125 years Darwin's ideas of natural selection have dominated the thinking of students of evolution (Darwin, 1859). It is only in the last 10-20 years that the other major Darwinian force of evolution-sexual selection (Darwin, 1871)-has provoked experimental biologists in a comparable way. There are several recent reviews that bear witness to this resurgence of interest (Campbell, 1972; Blum and Blum, 1979; O'Donald, 1980; Bateson, 1983). It has become clear that sexual selection may be implicated in the maintenance of many genetic polymorphisms, for example, inversion polymorphism in $D$. pseudoobscura (Spiess et al., 1966) and D. persimilis (Spiess and Spiess, 1967), colour polymorphism in ladybirds (O'Donald and Majerus, 1984), arctic skuas (O'Donald, 1983) and sticklebacks (Semler, 1971).

The sexual component of selection in systems of this type may be much greater than other components such as viability and fecundity (Prout, 1971). No study of selective pressures influencing a polymorphic system can be complete, therefore, without a consideration of sexual selection. Furthermore, polymorphic systems have a great poten- tial for investigating the operation of sexual selection, and its interaction with other components of selection. The pioneering work of O'Donald and his associates on Adalia bipunctata (O'Donald and Majerus, 1985) demonstrates that results of general importance can be obtained.

Previous work on the dipteran seaweed fly, Coelopa frigida, has shown that a variety of selective pressures act on an inversion polymorphism on chromosome I. Karyotypes differ in development time (Day et al., 1980), adult body size (Butlin et al., 1982a), viability (Butlin et al., 1984), longevity and fecundity (Butlin and Day, 1985). In laboratory tests where a female was able to mate with either a large or a small male, the large male sired her progeny in about 80 per cent of trials (Butlin et al., 1982a). The karyotypes of the males did not influence this result but in natural populations the association between karyotypes and male size was expected to produce an association between karyotype and male mating success. We have attempted to demonstrate such an association in nearly natural conditions and have found marked differences in male mating success. However, these differences do not appear to be associated with large male size. 


\section{GENERAL MATERIALS AND METHODS}

Animals were obtained from natural populations at two sites on the north-east coast of England: St. Mary's Island (Ordnance Survey map reference NZ 350753) and Whitburn (NZ 408614). Adults were collected using an insect aspirator, and larvae sampled using the "pre-adult" method described by Butlin et al. (1982a). The size of adults was estimated from the length of their wings (Butlin et al., 1982b). Their genotype at the alcohol dehydrogenase locus $(A d h)$-used as a marker for the chromosome I inversion (Day et al., 1982) was determined by starch gel electrophoresis (Butlin et al., 1982a), with the minor modification that 3-(4-,5-dimethylthiazolyl-2)-2,5-diphenyltetrazolium bromide (MTT) was used in the staining mixture instead of NBT.

\section{Pilot experiment}

The most direct way of studying mating success in wild populations of insects is to capture mating pairs. In seaweed flies mating usually takes less than 60 seconds, and animals are only rarely seen together as pairs. An additional problem is that mating occurs beneath the surface of the seaweed deposit, and disturbance of the weed results in the animals taking flight. In practice it has not proved possible to observe directly the mating behaviour of flies in their natural habitat. A less direct approach was necessary to study mating success. We carried out a pilot study to test the feasibility of identifying the genotypes of fathers inferred from the analysis of their progeny, and then comparing them with the genotypes of males available to be fathers. This technique has the advantage of measuring only successful copulations.
Males and females from a seaweed bed at St. Mary's Island were separated immediately after collection, 320 females placed individually in containers with fresh seaweed. The Adh genotype of each female that laid fertile eggs was determined, as were the genotypes of her progeny after they had grown to a sufficient size. The number of progeny examined was usually between 8 and 12 . Knowing the genotypes of the mother and her progeny allows us to infer the genotype of the father. In addition the genotypes and sizes of females that did not lay eggs, and of the males present in the original sample, were assessed.

Some females collected did not lay eggs (110), laid infertile eggs (172), or laid too few fertile eggs to infer unambiguously the father's genotype (5). Two females produced progeny that could not be accounted for in terms of a single male parent and so must have resulted from multiple insemination. In addition one female had been mated by a male with a very rare genotype $(A d h-D E)$. In this, and in all subsequent experiments, animals carrying the rare $A d h-A$ or $E$ alleles, as well as $C C$ homozygotes, have been omitted from the analysis. This amounts to an omission of less than 1 per cent of the data. From the original 320 females, we obtained information on 30 matings.

The genotypes of mothers, fathers, females and males, and the sizes of males, are given in table 1 . The mothers were a random sample of the females present, but the inferred fathers are significantly different from the males present, at least with respect to their Adh genotypes. This small sample of only 30 matings also provides no evidence that large males are more successful than small ones. Indeed, nearly half the matings seem to have involved the genotypic class of smallest mean size.

Table 1 Comparison of $A d h$ genotype frequencies in mothers, fathers, females and males (pilot experiment)

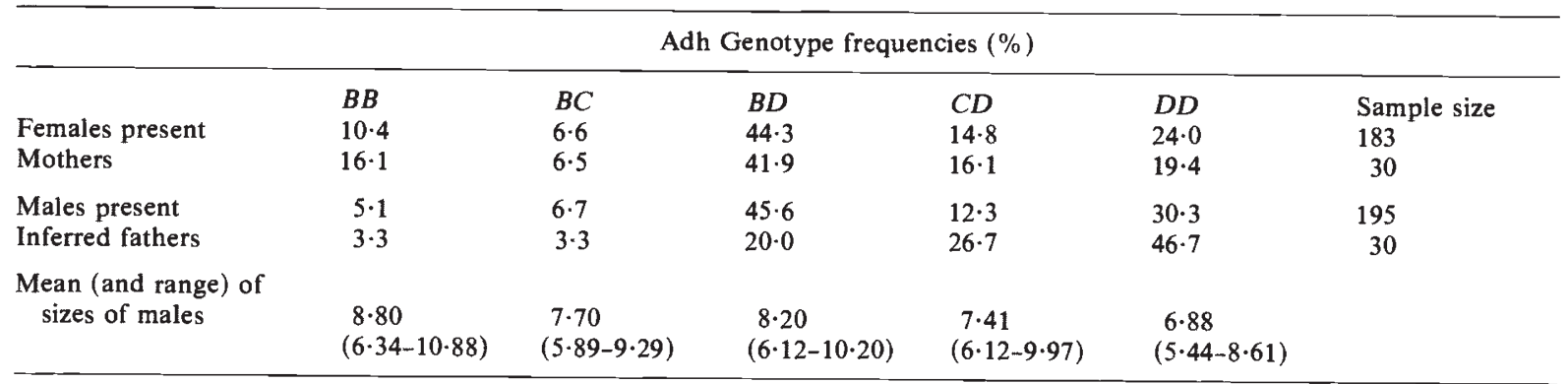

There is no significant difference between genotypes of mothers, and females available to be mothers $\left(\chi_{3}^{2}=1 \cdot 10, p=0 \cdot 78\right)$.

The inferred genotypes of fathers are significantly different from those of males available to be fathers $\left(\chi_{2}^{2}=9 \cdot 50, p=0 \cdot 009\right)$.

(Here and elsewhere $\chi^{2}$ values have been calculated using numbers rather than frequencies).

The sizes of males are expressed in graticule units ( 1 unit $=0.64 \mathrm{~mm}$ ). Mean sizes differ significantly between genotypes $\left(F_{4,194}=23.0 p<0.001\right)$. 
However, the main conclusion from this pilot experiment was that it was an extremely laborious and inefficient method of studying mating. A large proportion of the original females were infertile, presumably because of virginity or senility. In addition, the experiment can be criticised on the grounds that the males collected at the time of sampling were not necessarily representative of those available to be fathers at the time of mating. Differences in the longevity of males (Butlin and Day, 1985), and in particular, differences in the hatch sequence of the various genotypes (Day et al., 1980), result in genotype frequencies changing with time. Multiple insemination and the incorrect inference of the father's genotype (due to the limited number of progeny being scored) are further potential sources of inaccuracy.

\section{Main experiment}

In an attempt to overcome at least some of these shortcomings a different method of sampling was used. Instead of adults, samples of larvae, and of the seaweed in which they were living, were collected. These were then maintained at room temperature $\left(22^{\circ} \mathrm{C}\right)$ until the adults eclosed. They were left undisturbed to mate with whom they chose, and as often as they chose. After varying lengths of time, the adults were aspirated from the cages, and the males and females separated immediately. The males were frozen $\left(-25^{\circ} \mathrm{C}\right)$ for subsequent measurement and genotyping, and the females individually allowed to lay eggs. Females and their progeny were analysed as before.

We report here the results from three sets of samples each with slight modifications of the basic experimental design. The essential features of the three experiments were as follows:

Sample 1 Larvae were collected from St. Mary's Island in November 1979 and a single sample of adults taken just after the time of peak eclosion.

Sample 2 Larvae were collected in three replicate cages from St. Mary's Island in September 1981. Adults from the first cage (A) were sampled 1 day after the start of eclosion; the second cage (B) was sampled 3 days later, and the third cage (C) after a further 2 days. Adult sizes were not measured.

Sample 3 Larvae were collected in four replicate cages from Whitburn in November 1982. Adults from cages A, B, C and D were aspirated 2, 4, 6 and 8 days respectively, after adults were first seen. All males and females were measured. 12 progeny were used to infer paternity whenever possible.

\section{Mixed paternity}

Early studies on mating in Coelopa suggested that if a female is presented with first one male and then a second, it is the second male that sires the progeny (Thompson, 1951; Burnet, 1961). However if the two males are offered simultaneously then in about 15 per cent of cases the progeny are of mixed paternity (Butlin et al., $1982 a$ ). In the pilot study of wild caught flies, about 6 per cent $(2 / 33)$ of females appeared to have been multiply inseminated.

The most reliable estimates of mixed paternity emerged from samples $3 \mathrm{~A}, \mathrm{~B}, \mathrm{C}$ and $\mathrm{D}$ in which the largest number of progeny had been studied (usually 12 animals). From table 2 it can be seen that the proportion of progeny sired by more than one male varied from 3-9 per cent. However, this must be an underestimate, since some progeny must have been interpreted as resulting from a single father when in fact there were contributions from more than one male. For example, if an $A d h-B B$ mother had in fact been inseminated by both a $B B$ and a $B D$ male, then the inferred father would be a single $B D$ male. In other words, we were unable to distinguish between sampling error causing a deviation from Mendelian expectations, and distortions due to sperm mixture. However, if we make some simple assumptions then we can attempt to correct for this type of error. The assumptions and general formulae used in these calculations are given in the Appendix.

A further correction incorporated into the calculation of the expectations is sampling error

Table 2 Frequency of mixed paternity

\begin{tabular}{|c|c|c|}
\hline Sample & $\begin{array}{l}\text { Observed incidence } \\
\text { of mixed } \\
\text { paternity }(\%)\end{array}$ & $\begin{array}{l}\text { Calculated incidence } \\
\text { of multiple } \\
\text { insemination (per cent) } \\
\text { (observed and } \\
\text { undetected) }\end{array}$ \\
\hline Pilot & $6 \cdot 3$ & $40 \cdot 3$ \\
\hline 1 & $4 \cdot 8$ & $32 \cdot 2$ \\
\hline $2 \mathrm{~A}$ & 3.9 & $27 \cdot 1$ \\
\hline $2 B$ & $3 \cdot 9$ & $32 \cdot 2$ \\
\hline $2 \mathrm{C}$ & $6 \cdot 8$ & $57 \cdot 9$ \\
\hline $3 \mathrm{~A}$ & $3 \cdot 3$ & $18 \cdot 6$ \\
\hline $3 B$ & $6 \cdot 7$ & $43 \cdot 5$ \\
\hline $3 \mathrm{C}$ & $9 \cdot 1$ & $64 \cdot 4$ \\
\hline $3 \mathrm{D}$ & $6 \cdot 3$ & $32 \cdot 4$ \\
\hline
\end{tabular}

The "observed incidence" of mixed paternity is that proportion of broods found to possess three or more paternally derived alleles.

The "calculated incidence" is the estimated probability (per cent) of a female being inseminated by two males. (This is the value of $x$ in the model described in the Appendix). 
resulting from the limited number of progeny studied. We may by chance have failed to sample one genotype in the progeny and thereby incorrectly inferred the father's genotype. In practice this type of error is negligible (1-2 per cent) compared to that from mixed paternity. In general terms, the "corrected" expectations compensate for the under-estimation of homozygote frequencies, and the over-estimation of the frequencies of heterozygotes.

The calculated proportions of multipaternal families (whether detected as such or not) are given in table 2. It is clear from these data that multiple insemination is quite frequent; about $30-40$ per cent of broods are of mixed paternity. Not surprisingly it increases with time. Sample $3 \mathrm{~A}$ was taken first, and B, C and D at two day intervals thereafter. The same trend is seen in 2A, B and C. (Sample $3 \mathrm{D}$ was small-if 2 instead of 1 mixed mating had been observed, then the incidence would have been $64 \cdot 7$ per cent.)

Finally, these data indicate that the experimental technique grossly underestimated the frequency of mixed paternity. This must mean that the observed genotype frequencies among fathers are also distorted. For this reason the corrections outlined in the Appendix have been applied to generate modified expectations of paternal frequencies used in the next two sections.

\section{Are fathers a random sample of the males available to be fathers?}

There are two possible methods of analysing the data. The first involves calculating the sperm gene frequencies from the frequencies of genotypes in each brood. The frequency of paternally contributed genes can then be compared with the expected contribution calculated from the males available to be fathers. This is the approach used by Wilson (1981). An alternative method is to infer the father's genotype for each brood, and then to compare these genotype frequencies with those present in males available to be fathers. The first method is very sensitive to any viability differences between progeny individuals. The second method does not rely on the relative frequencies of genes in the progeny, but merely on their presence or absence. Since there is evidence for differences in viability among developing larvae (Collins, 1978; Butlin et al., 1982b; 1984), we have used the second method. It should be pointed out that the conclusions to which we come are not dependent on which method of analysis is used.
The observed and expected genotype frequencies among fathers for each sample are given in table 3 . It can be seen that there is a fairly general deficiency of fathers with the genotypes $B B, B C$, and $B D$, and an excess of fathers with $C D$ and $D D$ genotypes. The values of $\chi^{2}$ given derived from comparisons between the observed numbers of fathers and the numbers expected on the assumption of random mating. These expectations incorporate the corrections for mixed paternity and for the limited numbers of progeny examined. In all but two samples for which a valid statistical test could be carried out there is a very highly significant departure from randomness.

There are, of course, many ways in which these data could depart from randomness. The critical question is whether there is consistency in the patterns of non-randomness. If each male genotype in each sample is assigned a "success" rating (the ratio of observed/expected genotype frequencies in fathers), and these ratings are ranked within samples, then the nine series of ranks can be compared. From Kendall's coefficient of concordance $(w=0.768, p<0.001$; see Sokal and Rohlf, 1981), it is clear that animals in the different samples are behaving in a homogeneous fashion.

We are particularly impressed by the consistent success of $C D$ males as fathers (more than three times the expected), and the lack of success of $B B$ males (about one quarter the expected).

These differences in genotype frequencies between inferred and expected fathers could be generated in several ways. They could result from real differences in mating success between genotypes, in which case they would probably be mediated by some phenotypic character associated with the chromosome I inversion. The obvious candidate in view of previous work (Butlin et al., $1982 a$ ) would be size, but the inversion contains many genes, so that a wide range of possible characters exists. The differences could be a result of the variation in development time between genotypes (Day et al., 1980) with no variation in mating success amongst mature males available at the time of mating. Finally, they could be an artefact of the procedures used to infer paternity and to calculate the expected distribution of fathers. The example given in the Appendix demonstrates that, despite the inferred high frequency of multiple insemination, the corrected expectations differ little from expectations based simply on the genotype frequencies amongst available males. We can envisage no way in which our methods of analysis could generate the large and consistent deviations observed. 
Table 3 Comparison of observed and expected Adh genotype frequencies (percentages) among fathers

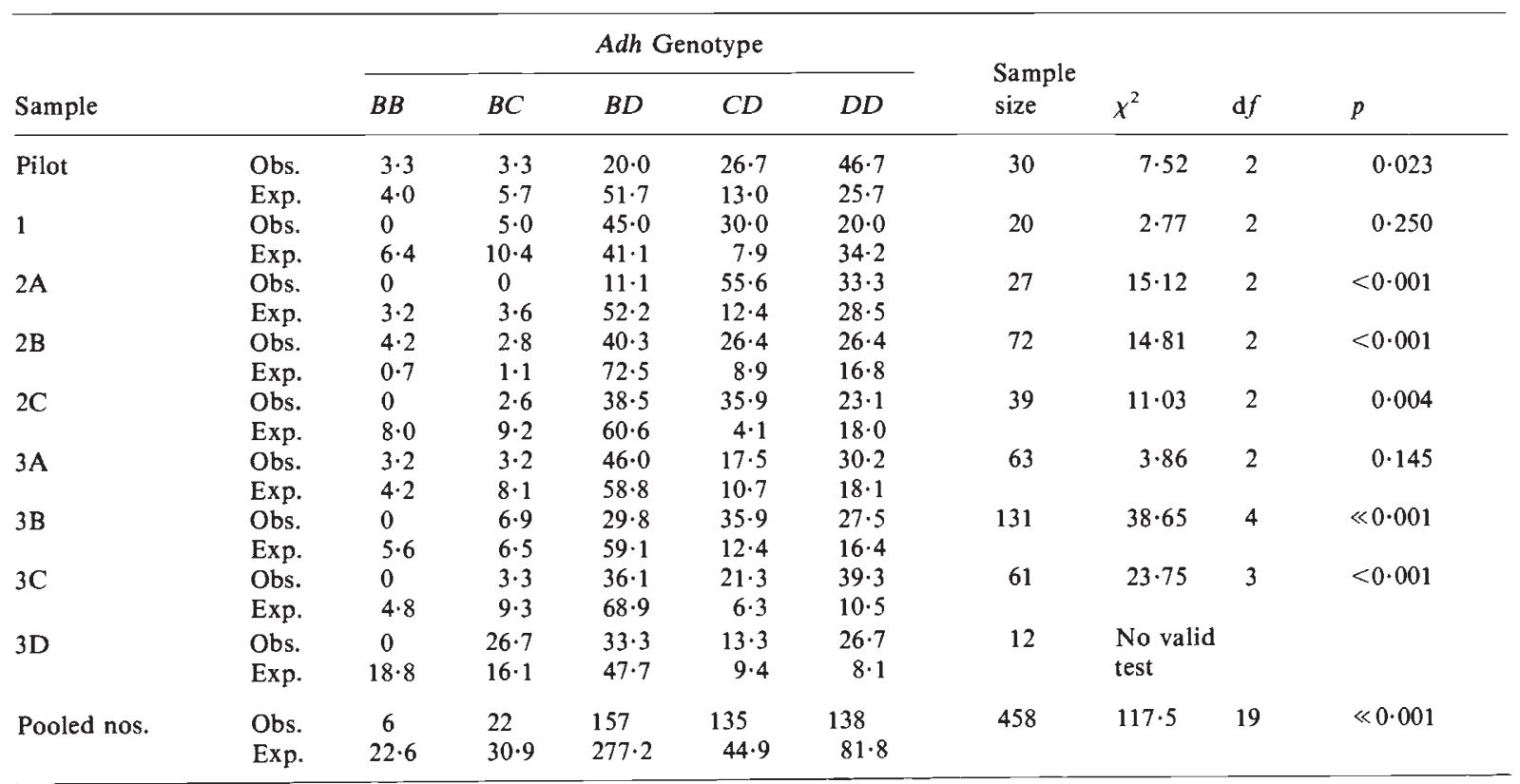

The "observed" genotype frequencies are based on inferred paternity from the analysis of maternal and progeny genotypes. The "expected" genotype frequencies are based on the males available to be fathers and incorporate the corrections for multi-paternity and sampling error of progeny (see Appendix).

\section{Is the reproductive success of males associated with their size?}

The sizes of males were measured in six of the samples. We have used the same simple measure of reproductive success as before-the ratio of observed/expected genotype frequencies in fathers (see table 4). If both variables are ranked within each of the six experiments, none of the rank correlation coefficients is statistically significant. This is hardly surprising since there are only five genotypes available for ranking. However, we can combine the data from the six samples to yield 30 pairs of ranks. The largest genotype in each sample is ranked equally, but each has its own success ranking from 1-5. Similarly, the second largest, third largest, fourth largest, and finally, the smallest, each has its own success ranking. This allows an assessment of the general correlation between size and success in spite of the fact that there are obvious differences in the sizes of animals between samples. This analysis reveals a strong negative association between male size and reproductive success $\left(r_{s}=-0.570, p=0.001\right)$. Furthermore, there is highly significant concordance between the samples in the relationship between size and success $(w=0.65, p=0.004)$. In other words, the mean reproductive success of males of a given genotype increases as the mean size of the genotype decreases, and does so in a very consistent way. Clearly the variation in mating success between genotypes in these experiments cannot be explained in terms of the genotype-independent advantage to large males observed in the laboratory (Butlin et al., 1982a). If the present results are accepted as representative of the real pattern of mating success in natural populations, then the previous laboratory experiments must either have missed a genotypic effect large enough to obscure the advantage to large males, or it must have artificially induced the success of large males. Note that the differences in mean size between genotypes in these samples are similar to the size differences between pairs of males in the laboratory studies. However, the size variation within each genotype is large.

\section{Is the time of eclosion associated with male reproductive success?}

There is a strong association between the time taken for seaweed flies to develop from egg to adulthood, and the chromosome I inversion (Day et al., 1982). Development time is also closely associated with adult size (Butlin and Day, 1984) 
Table 4 Reproductive success and mean sizes of males

\begin{tabular}{|c|c|c|c|c|c|c|}
\hline \multirow[b]{2}{*}{ Sample } & & \multicolumn{5}{|c|}{ Adh Genotype } \\
\hline & & $B B$ & $B C$ & $B D$ & $C D$ & $D D$ \\
\hline \multirow[t]{2}{*}{ Pilot } & Size & $7 \cdot 72$ & $6 \cdot 76$ & $7 \cdot 20$ & $6 \cdot 50$ & $6 \cdot 04$ \\
\hline & Success & $0 \cdot 83$ & 0.58 & $0 \cdot 39$ & $2 \cdot 05$ & $1 \cdot 82$ \\
\hline \multirow[t]{2}{*}{1} & Size & $8 \cdot 82$ & $8 \cdot 18$ & $7 \cdot 66$ & $6 \cdot 16$ & 5.92 \\
\hline & Success & 0 & 0.48 & 1.09 & $3 \cdot 80$ & 0.58 \\
\hline \multirow[t]{2}{*}{$3 A$} & Size & $10 \cdot 05$ & $8 \cdot 90$ & $9 \cdot 19$ & $8 \cdot 74$ & $7 \cdot 39$ \\
\hline & Success & 0.76 & 0.40 & $0 \cdot 78$ & $1 \cdot 64$ & $1 \cdot 67$ \\
\hline \multirow[t]{2}{*}{$3 B$} & Size & $9 \cdot 70$ & 8.97 & $8 \cdot 71$ & $8 \cdot 25$ & $7 \cdot 24$ \\
\hline & Success & 0 & 1.06 & 0.50 & $2 \cdot 90$ & 1.68 \\
\hline \multirow[t]{2}{*}{$3 \mathrm{C}$} & Size & $10 \cdot 16$ & $9 \cdot 53$ & $9 \cdot 41$ & $8 \cdot 36$ & $7 \cdot 75$ \\
\hline & Success & 0 & $0 \cdot 35$ & 0.52 & $3 \cdot 38$ & $3 \cdot 74$ \\
\hline \multirow[t]{2}{*}{$3 \mathrm{D}$} & Size & $9 \cdot 80$ & $9 \cdot 05$ & $8 \cdot 57$ & $7 \cdot 98$ & $7 \cdot 17$ \\
\hline & Success & 0 & $1 \cdot 66$ & $0 \cdot 70$ & 1.41 & $3 \cdot 30$ \\
\hline
\end{tabular}

Reproductive success is the ratio of observed frequencies of fathers to expected frequences (based on the observed frequencies of males available to be fathers incorporating the corrections for multi-paternity and limited sampling of progeny-see Appendix). The mean size of males are expressed in graticule units ( 1 unit $=0.64 \mathrm{~mm}$ )

such that genotypes which develop rapidly produce small adults. This could have affected our results because the males apparently available may have contained some immature individuals. In this case there could be a bias towards the later emerging genotypes ( $B B, B C$ and $B D$ ) relative to those actually capable of fertilising females. This would be aggravated by the fact that the females in a sample may have mated some time before the sample was taken. However, in natural populations, eggs are not laid synchronously so that the emergence of the different genotypes is far from being strictly sequential. Butlin (1983) found that the $A d h-B$ frequency in male flies emerging from a sample similar to those used in experiments 1 , 2 and 3 , changed from $0-0.30$ in the first 2 days of emergence, to 0.37 in 4 days, and very little subsequently. The females emerged throughout this period with no change in genotype frequency. The changes in expected frequencies (table 3 ) show the same sort of pattern in experiments 2 and 3 with only a slight increase in $A d h-B$ and decrease in $A d h-D$ between cages.

Males and females mate first about 24 hours after eclosion and thereafter both sexes mate repeatedly (Butlin, 1983). That several males can contribute to a female's offspring is apparent in the present results and from previous laboratory work (Butlin et al., 1982a).

Combining these observations it is clear that development rate variation is unlikely to have a major effect on our results. Any effect it did have would be most pronounced in the early samples (2A and $3 \mathrm{~A}$ ) which should show higher mating success for rapidly developing genotypes $(C D$ and $D D$ ) than subsequent samples. In fact there is no consistent trend in this direction (table 3 ).

It remains possible that rapid development or early emergence contribute in some indirect way to the mating success of the $C D$ and $D D$ genotypes. However, the observed variation in mating success cannot be an artefact of changing genotype frequencies over the emergence period.

\section{Is the reproductive success of females associated with their Adh genotype and size?}

Are females that laid fertile eggs a random sample of the females present? For six samples the answer is positive (see table 5). However, there is a significant departure from randomness in two samples, where there is either an excess of $B B$ 's or a deficiency of $D D$ 's among the mothers. Indeed in most samples there is a tendency for the reproductive success of $B B$ 's and $B C$ 's to be above average and for $D D$ 's to be below average.

In all four samples in which female sizes were measured there is no correlation of female size with reproductive success $\left(r_{s}=0.014, p>0.9\right)$. There is a tendency for the larger females to be more fertile but this does not even approach statistical significance. However, when the sizes of females that laid eggs are compared (within each genotype) with those that did not, in nearly every case (19 out of 20 ) the fertile females were larger (data not shown). At the time of sampling it may only be the largest females that still have unlaid eggs available to be analysed in this experiment. This may be the explanation-admittedly a rather trivial one-for the apparent reproductive success of $B B$ and $B C$ females (the largest and youngest genotypes), and the lack of success of $D D$ 's (the smallest and oldest genotype).

In summary, we have presented evidence that under reasonably natural conditions, there are highly significant differences in the reproductive success of males with respect to their $A d h$ genotype. There is a negative correlation between mean male size and mating success of genotypic classes. The results could be influenced by variation in emergence time. This effect is expected to be small relative to the observed variation in mating success, and there is no evidence for its occurrence in the successive samples of experiments 2 and 3 .

The reproductive success of females is usually random with respect to both $A d h$ genotype and 
Table 5 Adh Genotype Frequencies among Females and Mothers

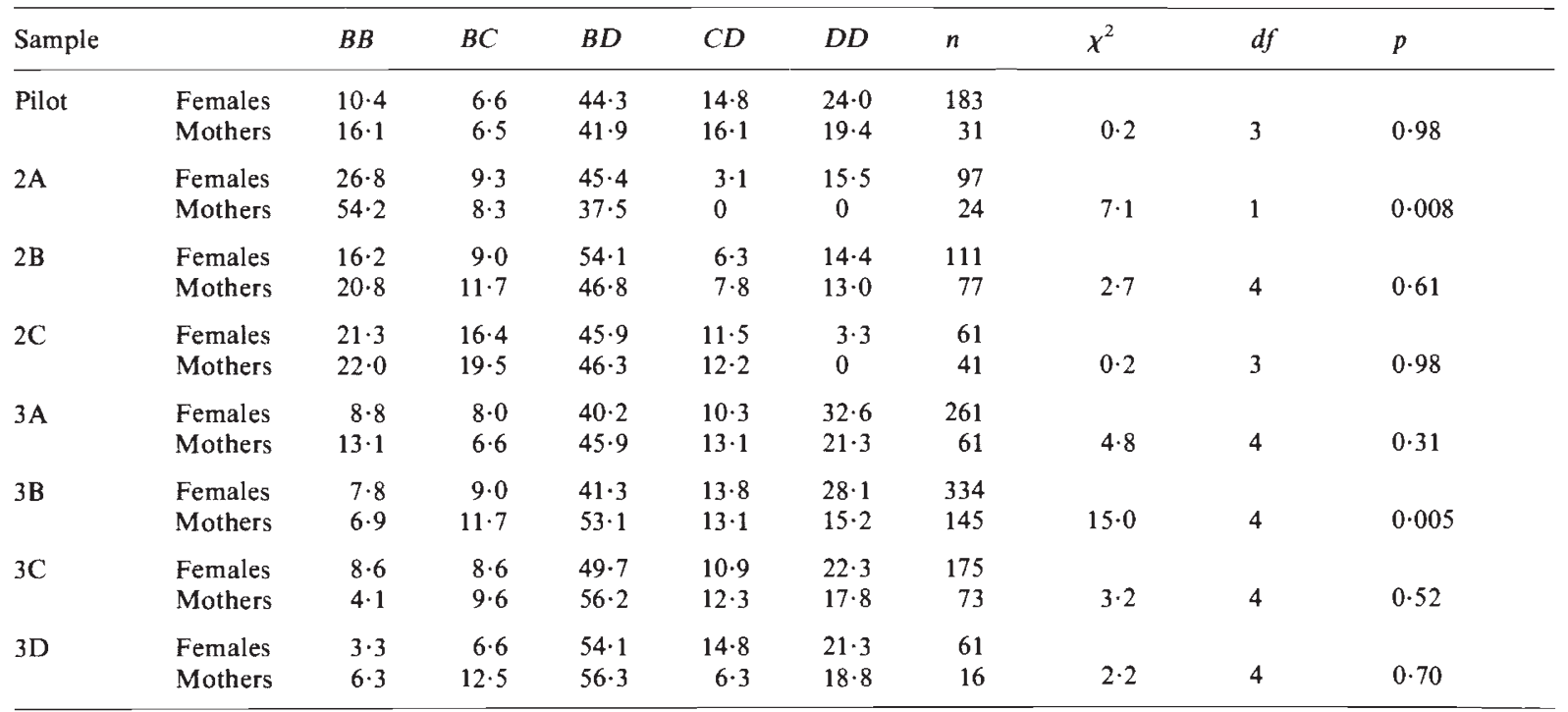

size. The departures from randomness that were observed in two samples, may well be a consequence of only the larger and younger females still having eggs available to be analysed.

\section{DISCUSSION}

In laboratory experiments large male seaweed flies father most of the progeny with inversion karyotype apparently having little influence on mating success (Butlin et al., 1982a). Under more natural conditions there is variation between genotypes in male success correlated with the average sizes of genotypes, but small genotypes are the most successful. The reasons for this difference must lie in the design of the two experiments and need to be resolved before the influence of sexual selection on the chromosome I inversion polymorphism can be analysed.

Variation in emergence times of the genotypes from natural seaweed deposits was a potential confounding factor in the present set of experiments. We have argued that its effect on mating success was likely to be small, and in any case would have been confined to the early samples. In fact the pattern of mating success is strikingly consistent over all samples.

The conditions under which mating occurred in the two sets of experiments were very different. In the laboratory trials, individual 3-day-old virgin females were confined for 24 hours in small vials with two males. By contrast, in the more natural experiments described here, hundreds of flies with approximately equal numbers of males and females occupied large and complex habitats, and females probably remained virgin for no more than 24 hours. It is possible that the females in the laboratory experiment were exposed to many more mating attempts and, being unable to escape or hide, mated many times. This would almost certainly have been encouraged by the females' highly receptive state (being 3-day-old virgins), and could have favoured large males which are known to be able to mate more often than small males (Butlin and Day, 1985). In such a situation any female preference for particular male genotypes could have been masked, especially as there is sperm mixing (Butlin, 1983). Females in the "natural population" experiments would have had much more opportunity to exercise preferences or to avoid remating by retreating to crevices in the seaweed. In summary, we consider that the discrepant results obtained from the two experiments are a consequence of different levels of sex starvation, and habitat complexity.

Nevertheless, other factors may also be involved. The laboratory study involved only two genotypes, $B B$ (chromosomally $\alpha \alpha$ ) and $D D(\beta \beta)$, and the flies were from lines that had experienced some inbreeding and adaptation to laboratory culture conditions. In the population experiments all genotypes were present in natural frequencies with $B D(\alpha \beta)$ and $D D$ being the most common genotypes. This could certainly be a reason for a stronger genotypic effect observed in the latter 
case. The physical conditions at the time of mating, such as light, temperature and the decomposed nature of the seaweed, were also different.

We consider the present experiments come close to revealing the pattern of mating success in natural populations. We therefore suggest that the success of large males in the laboratory trials was influenced by the unusual conditions of the experiment. It remains possible that within genotypes there is an advantage to large males in natural conditions as has been described in many insect species (e.g., Ewing, 1964; Monclus and Prevosti, 1971; Thornhill, 1976, 1983; Rutowski, 1980; Baldwin and Bryant, 1981; Boggs, 1981; Woodhead, 1981; Johnson, 1982; McLain, 1982; O'Neill and Evans, 1983; Ward, 1983).

At present we do not know the behavioural basis for variation in mating success between genotypes. We have observed no aggression between males in the vicinity of females, and no long-distance courtship behaviour. The male and female only appear to interact after mounting and before copulation, and it is not uncommon for females to dislodge males at this stage. The possibility of female choice therefore exists. Small male size could possibly be the proximate character chosen by females but many others could be involved. The chromosome I inversion includes nearly 10 per cent of the genome and may therefore influence numerous phenotypic traits. If any of these was involved in mate choice an indirect association between mating success and both genotype and size would result. It is possible that female behaviour varies with chromosome I genotype, producing departures from random mating as well as variation in mating success. This issue is considered in the accompanying paper (Day and Butlin, 1987). Mating behaviour in seaweed flies is currently under investigation.

All natural populations of seaweed flies so far studied in the British Isles are polymorphic for the chromosome I inversion and the associated $A d h$ alleles (Butlin et al., 1982b; Butlin, 1983). The $\alpha$ frequency is usually in the range $0 \cdot 3-0.4$ but there is evidence for a seasonal cycle with $\alpha$ increasing in the summer and decreasing in the winter (Butlin, 1983; Day et al., in prep.). The polymorphism is maintained by heterokaryotypic advantage in viability (Butlin et al., 1984) but inversion frequencies must also be influenced by development times and variation in mating success. Clearly, an improved understanding of the sexual component of selection in seaweed flies is needed before we can satisfactorily account for this polymorphism involving a substantial fraction of the complete genome.
Acknowledgements This work was supported by a N.E.R.C. grant to T. H. Day, and by an S.E.R.C. studentship to R. K. Butlin. Their help is greatly appreciated.

\section{REFERENCES}

BALDWIN, F. T. AND BRYANT, E. H. 1981. Effect of size upon mating performance within geographic strains of the housefly, Musca domestica L. Evolution, 35, 1134-1141.

BATESON, P. 1983. Mate choice. Cambridge University Press, Cambridge.

BLUM, M. S. AND BLUM, N. A. 1979. Sexual Selection and Reproductive Competition in Insects. Academic Press, New York.

BOGGS, C. L. 1981. Selection pressures affecting male nutrient investment at mating in Heliconiine butterfies. Evolution, $35,931-940$.

BURNET, B. 1961. On the distribution of recessive embryonic lethals in a natural population of Coelopa frigida. Genet. Res., 2, 249-271.

BUTLIN, R. K. 1983. The maintenance of an inversion polymorphism in Coelopa frigida. PhD Thesis, University of Nottingham.

BUTLIN, R. K. AND DAY, T. H. 1985. Adult size, longevity and fecundity in the seaweed fly, Coelopa frigida. Heredity, 54, 107-110.

BUTLIN, R. K., READ, I. L., AND DAY, T. H. 1982a. The effects of a chromosomal inversion on adult size and male mating success in the seaweed fly, Coelopa frigida. Heredity, 49 , $51-62$.

BUTLIN, R. K., COLLINS, P. M., SKEVINGTON, S. J., AND DAY, T. H. 1982 b. Genetic variation at the alcohol dehydrogenase locus in natural populations of Coelopa frigida. Heredity, $48,45-55$.

BUTLIN, R. K., COLLINS, P. M. AND DAY, T. H. 1984. The effect of larval density on an inversion polymorphism in the seaweed fly, Coelopa frigida. Heredity, 52, 415-423.

CAMPBELL, B. G. 1972. Sexual Selection and the Descent of Man. Heinemann Educational Books Ltd., London.

COLliNS, P. M. 1978. Studies on genetic polymorphism in Coelopa frigida. PhD Thesis, University of Nottingham.

DAR WIN, C. 1859. On the Origin of Species by Means of Natural Selection. Murray, London.

DARWIN, C. 1871. The Descent of Man and Selection in Relation to Sex. Murray, London.

DAY, T. H. AND BUTLIN, R. K. 1987. Non-random mating in natural populations of the seaweed fly, Coelopa frigida. Heredity, 58, 213-220.

DAY, T. H., DOBSON, T., HILlIER, P. C., PARKIN, D. T. AND CLARKE, B. C. 1980. Different rates of development associated with the alcohol dehydrogenase locus in the seaweed fiy, Coelopa frigida. Heredity, 44, 321-326.

DAY, T. H., DOBSON, T., HILLIER, P. C., PARKIN, D. T. AND CLARKE, B. C. 1982. Associations of enzymic and chromosomal polymorphisms in the seaweed fly, Coelopa frigida. Heredity, 48, 35-44.

EWING, A. W. 1964. The influence of wing area on the courtship behaviour of Drosophila melanogaster. Anim. Behav., 12, 316-320.

JOHNSON, L. K. 1982. Sexual selection in a brentid weevil. Evolution, 36, 251-262.

MCLAIN, D. K. 1982. Density dependent sexual selection and positive phenotypic assortative mating in natural populations of the soldier beetle, Chauliognathus pennsylvanicus. Evolution, 36, 1227-1235. 
MONClUS, M. AND PREVOSTI, A. 1971. The relationship between mating speed and wing length in Drosophila subobscura. Evolution, 25, 214-217.

O'Donald, P. 1980. Genetic Models of Sexual Selection. Cambridge University Press, Cambridge.

O'Donald, P. 1983. The Arctic Skua. Cambridge University Press, Cambridge.

O'DONALD, P. AND MAJERUS, M. E. N. 1984. Polymorphism of melamic ladybirds maintained by frequency dependent sexual selection. Biol. J. Linn. Soc., 23, 101-111.

O'DONALD, P. AND MAJERUS, M. E. N. 1985. Sexual selection and the evolution of preferential mating in ladybirds. 1. Selection for high and low lines of female preference. Heredity, 55, 401-412.

O'NEILL, K. M. AND EVANS, H. E. 1983. Alternative male mating tactics in Bembecinus quinquespinosus (Hymenoptera; Sphecidae): Correlations with size and colour variation. Behav. Ecol. Sociobiol., 14, 39-46.

PROUT, T. 1971. The relation between fitness components and population prediction in Drosophila. I. The estimation of fitness components. Genetics, 68, 153-229.

RUTOWSKI, R. L. 1980. Courtship solicitation by females of the checkered white butterfly, Pieris protodice. Behav. Ecol. Sociobiol., 7, 113-117.

SEMLER, D. E. 1971. Some aspects of adaptation in a polymorphism for breeding colours in the threespine stickleback (Gasterosteus aculeatus). J Zool. Lond., 165, 291-302.

SOKAL, R. R. AND ROHLF, F. J. 1981. Biometry, 2nd edn. W. H. Freeman and Co., San Francisco.

SPIESS, E. B., LANGAR, B. AND SPIESS, L. D. 1966. Mating control by gene arrangements in Drosophila pseudoobscura. Genetics, 54, 1139-1149.

SPIESS, E. B. AND SPIESS, L. D. 1967. Mating propensity, chromosomal polymorphism and dependent conditions in Drosophila persimilis. Evolution, 21, 672-678.

THOMPSON, U. 1951. Studies on the genetics and ecology of Coelopa frigida (Fab). PhD Thesis, University of Durham.

THORNHILL, R. 1976. Sexual selection and nuptual feeding behaviour in Bittacus apicalis (Insecta: Mecoptera). Am Nat., 110, 529-548.

THORNHILL, R. 1983. Cryptic female choice and its implications in the scorpion-fly Harpobittacus nigriceps. Am Nat., 122, 765-788.

WARD, P. I. 1983. The effects of size on the mating behaviour of the dung fly Sepsis cynipsea. Behav. Ecol. Sociobiol., 13, 75-80.

WILSON, J. 1981. Estimating the degree of polyandry in natural populations. Evolution, 35, 664-673.

WOODHEAD, A. P. 1981. Female dry weight and female choice in Chauliognathus pennsylvanicus. Evolution, 35, 192-193.

\section{APPENDIX}

\section{Calculation of expected frequencies among fathers}

We describe here the modifications to expectations resulting from multiple paternity and sampling error of progeny. We make the following assumptions:

(a) There are no fecundity differences between males, nor between females.

(b) The probability of a mating between any two genotypes is determined solely by the genotype frequencies in the two sexes. Thus the probability of a mating between a $B B$ female and a $B D$ male is the product of the frequency of $B B$ 's among females and the frequency of $B D$ 's among males.

(c) The probability of a second or subsequent mating is determined solely by the relevant genotype frequencies, and is independent of a previous mating.

(d) There are no differences in the survival of zygotes related to their $A d h$ genotype.

(e) Broods resulting from three or more inseminations are not considered. While these assumptions may not be met precisely, they are necessary for the construction of a workable model. Small departures are unlikely to affect the conclusions.

Let $p, q, r, s$ and $t$ be the observed frequencies among the males available to be fathers, of $A d h$ genotypes $B B, B C, B D, C D$, and $D D$ respectively; and $p, q, r, s$ and $t$ be the observed frequencies among mothers; and $x$ be the probability of a given female being inseminated by two males.

The expected frequencies among fathers (exp $\mathrm{f} B B, \exp \mathrm{f} B C$ etc) have two componentsone derived from unipaternal broods, and the other from bi-paternal broods:

$$
\begin{aligned}
& \exp \mathrm{f} B B=(1-x) p+x p^{2} \\
& \exp \mathrm{f} B C=(1-x) q+x\left(q^{2}+2 p q\right) \\
& \exp \mathrm{f} B D=(1-x) r+x\left(r^{2}+2 r p+2 p t+2 r t\right) \\
& \exp \mathrm{f} C D=(1-x) s+x\left(s^{2}+2 s t\right) \\
& \exp \mathrm{f} D D=(1-x) t+x t^{2}
\end{aligned}
$$

and the frequency of multi-paternal broods expected to be detected is:

$$
\mathrm{f} M P=2 x(p s+q r+q s+q t+r s)
$$

In order to correct these expectations for sampling error resulting from only a limited number of each brood being sampled, we have considered the Mendelian ratios in the progeny for every genotype of the mother, and every combination of two males. Assuming that 10 animals were examined from each brood, it is then easy to calculate the probability of each type of double mating being scored in any particular way. For example, if a $C D$ female was inseminated by both a $B C$ and a $C D$ male, then the probability that the inferred father will be $B C$ is $0 \cdot 234$. (Among the 10 progeny there must be at least one $B C$ or $B D$, and at least one $C C$, and no $D D$ s.) By similar reasoning the probability that the inferred father will be a $B D$ is 0.039 , and that it will be $C D$ is also 0.039 . The chances that the mating will be scored as multi-paternal is 0.656 . 
(In this case among the progeny there must be at least one each of $C C, D D$ and either $B C$ or $B D$.) It is further assumed that the probability of such a mating occurring is simply the product of the mother's genotype frequency and of the frequencies of the two males involved. In the example given above the probability of these three animals being involved is the product of $s, q$, and $s$.

The expected frequency among the fathers is then summed over all possible combinations of matings. This leads to corrected expressions for $\exp \mathrm{f} B B$, exp $\mathrm{f} B C$ etc and for $\mathrm{f} M P$. By substituting the observed frequency of multi-paternal broods in the equation for $\mathrm{f} M P$, an estimate of $x$, the actual frequency of double insemination, can be obtained. This can then be used to obtain values for the expected frequencies of each genotype.

The general effect of these corrections is to reduce the expectation for homozygotes, and increase them for heterozygotes. In practice, the corrections for progeny sampling error is small (approximately 1-2 per cent), but the correction for multiple insemination can be of greater consequence. An example will serve to illustrate the effect of the corrections. The following table shows the expectations of the fathers' frequencies in $3 \mathrm{~B}$ (the largest sample) when calculated simply from the frequencies in males, and when calculated with corrections for multi-paternity and progeny sampling error.
Inferred nos. of fathers

Expected nos. of fathers (without correction)

Expected nos. of fathers (with correction)

\begin{tabular}{cllllll}
\multicolumn{7}{c}{ Adh Genotype } \\
\cline { 1 - 1 } & $B C$ & $B D$ & $C D$ & $D D$ & $\chi_{4}^{2}$ & $p$ \\
0 & 9 & 39 & 47 & 36 & & \\
$10 \cdot 1$ & 8.8 & 69.4 & 15.7 & 27.1 & 34.9 & $\ll 0.001$ \\
$7 \cdot 3$ & 8.5 & 77.4 & 16.2 & 21.5 & 38.1 & $\ll 0.001$
\end{tabular}

The observed data deviate very significantly from both sets of expectations, but the comparison with the corrected expectations yields a slightly larger value of $\chi^{2}$. 